\title{
Detection of piroplasms infection in sheep, dogs and hedgehogs in Central China
}

\author{
Zhuo Chen ${ }^{1,2}$, Qin Liu ${ }^{1,2^{*}}$, Feng-Chao Jiao ${ }^{3}$, Bian-Li Xu ${ }^{4}$ and Xiao-Nong Zhou ${ }^{1,2^{*}}$
}

\begin{abstract}
Background: Piroplasms are kinds of tick-borne parasitic apicomplexan protozoa, which are detrimental to humans and animals in tropical and subtropical areas around the world. Up until now, there has been a limited amount of reliable information available about the prevalence of piroplasms infections in wild animals in China. Therefore, we have investigated the infections of Babesia and Theileria species in both domestic and wild animals in Xinyang city, Henan province, where tick-borne diseases have recently been reported. This study aims to analyze the distribution patterns of piroplasms infections in animals, and assess their potential threat to humans in Central China.

Methods: Blood samples were collected from sheep, dogs and hedgehogs in two regions, including Shihe District and Luoshan County, of Xinyang city, Henan province from August to December 2012. Babesia spp. and Theileria spp. were detected by polymerase chain reaction (PCR) and identified by sequencing and phylogenetic analysis. Moreover, the characteristics of detected piroplasms in different animal hosts were compared between the two study regions.
\end{abstract}

Results: A total of 227 blood samples were collected from 73 sheep, two dogs and 152 hedgehogs. Babesia spp. was only detected in the two dogs. Theileria spp. was detected both in the sheep and the hedgehogs, and the total positive rate of Theileria spp. in the sheep and the hedgehogs was $57.53 \%$ and $13.82 \%$, respectively. Sequencing and phylogenetic analysis revealed that the Theileria spp. detected in the sheep and the hedgehogs were very close to T. lunwenshuni cloned from a small ruminant and Theileria spp. isolated from a febrile hospitalized patient in China.

Conclusion: Babesia and Theileria infections were detected in both domestic and wild animals in Xinyang city, Henan province in Central China, thus warranting further studies in these regions.

Keywords: Babesia spp, Theileria spp, Dogs, Sheep, Hedgehogs, China

\section{Multilingual abstracts}

Please see Additional file 1 for translations of the abstract into the six official working languages of the United Nations.

\section{Background}

Piroplasmosis is a tick-borne disease which is caused by infection with apicomplexan protozoa, such as the Babesia or the Theileria species. These protozoan parasites are transmitted by vector ticks and can infect many different

\footnotetext{
*Correspondence: liuqin0901@sohu.com; zhouxn1@chinacdc.cn 'National Institute of Parasitic Diseases, Chinese Center for Disease Control and Prevention, Shanghai 200025, People's Republic of China ${ }^{2}$ WHO Collaborative Center for Malaria, Schistosomiasis and Filariasis; Key Laboratory of Parasite and Vector Biology, Ministry of Health, Shanghai 200025, People's Republic of China

Full list of author information is available at the end of the article
}

species of domestic and wild animals. Some species of the protozoan parasites are also pathogenic to humans [1-3].

Theileriosis and babesiosis are distributed widely in China. For instance, B. caballi and T. equi were detected in horses mainly in the northeastern and northwestern parts of China [4-6]. T. buffeli, T. sergenti, B. bigemina, $B$. bovis, B. ovata and B. orientalis were detected in cattle and buffalo in Central and southern China [7-9]. T. ovis, T. uilenbergi, T. luwenshuni and B. motasi were detected in sheep and goats mainly in Gansu, Ningxia, Qinghai, Henan, Inner Mongolia and Jilin provinces [10-13]. B. gibsoni and B. canis vogeli were detected in dogs in Jiangsu, Chongqing, Guangdong, Guangxi, Hainan and Zhejiang provinces $[14,15]$. B. microti infections were reported in animals and humans in the northeastern and southern parts of China $[16,17]$. However, only a limited amount of information is available on the prevalence of 
piroplasmosis in wild animals in China. There are only a few reports which documented small rodents infected with $B$. microti in Taiwan, Beijing, Zhejiang and Heilongjiang provinces [18-21] and Sika deer infected with Theileria spp. in Hubei province [22]. Some species of piroplasms may lead to high mortality and low productivity to susceptible animals, for instance, $T$. annulata was highly pathogenic to cattle [23]. Some other species, however, seemed to be benign to the host animals, and most of the infected animals did not show any clinical symptoms or signs of diseases [24].

Babesia spp., such as $B$. microti and B. divergens, are the major pathogenetic species of piroplasms causing human infections. However, some Theileria species can also cause severe acute diseases in humans as human theileriosis $[25,26]$. Previous research reported that $2.3 \%$ of 432 samples (from volunteers) were detected positive for Theileria equi antigens by indirect fluorescent antibody test (IFAT) [26]. Recently, a febrile hospitalized patient in Suizhou city of Hubei province was reported to be infected by a Theileria species (18S rRNA GenBank accession number HQ844673) by Liu et al. [27] which was very close to $T$. lunwenshuni.

Although piroplasmosis is one of the most prevalent diseases of domestic animals, the epidemiology and transmission characteristics among vector ticks and animal hosts are still unclear, and the situation would become even more complex if wild animals were acting as the natural reservoirs.

Thus, we investigated the infections of Babesia spp. and Theileria spp. in both domestic and wild animals in Xinyang city where tick borne diseases were recently reported, in order to analyze the distribution patterns of piroplasms infections in animals and assess their potential threat to humans in Central China.

\section{Methods}

\section{Ethical clearance}

The National Institute of Parasitic Diseases, Chinese Center for Disease Control and Prevention issued the ethical and institutional approval documents for this study.

\section{Sample collection}

This study was carried out in Luoshan County and Shihe District of Xinyang city in Henan province from August to December 2012 (see Figure 1). The sampling sites were determined by a method of random grid sampling, which was performed using ArcGIS. A total of 227 blood samples were collected from 152 hedgehogs, 73 sheep and two dogs. Blood samples from the two dogs were collected from Shihe District. Detailed information on the numbers of blood samples collected from hedgehogs and sheep in each region is shown in Table 1. In this study, the veterinarians rescued the hedgehogs which were caught by the local people, and checked their health conditions. A very small amount of blood (less than $200 \mu \mathrm{l}$ ) was drawn from each animal, an amount that is harmless to the animal's health. After this, the hedgehogs were released into their natural habitat. Blood samples were collected into an EDTA containing tube and kept frozen at $-20^{\circ} \mathrm{C}$ until further processing.

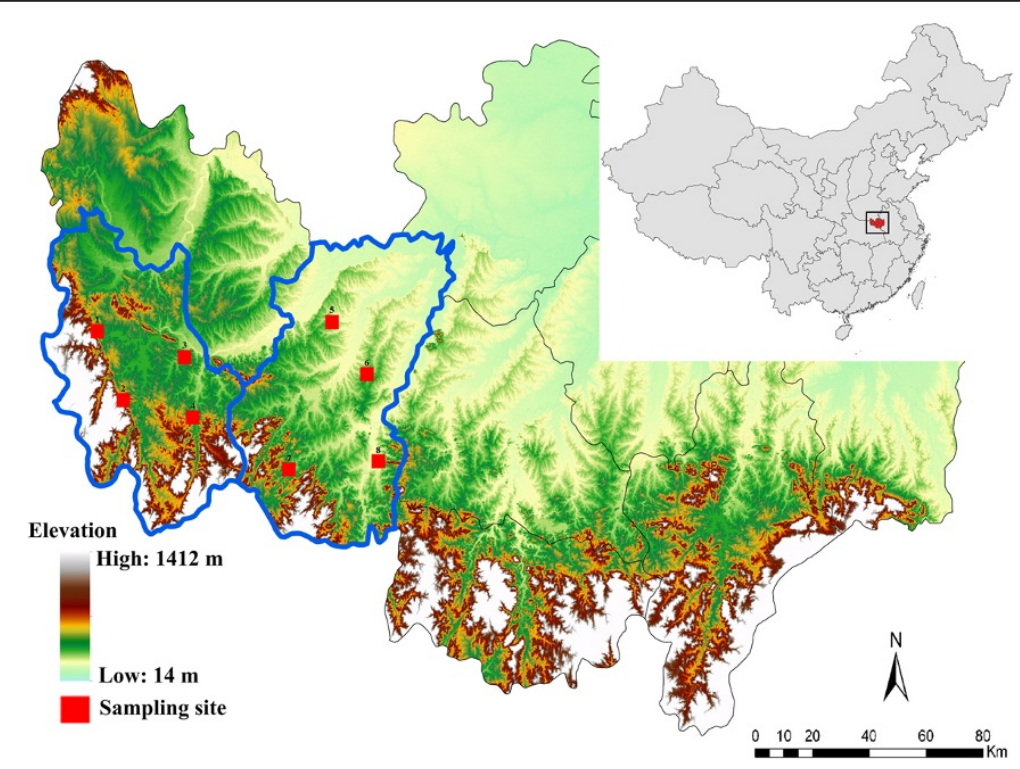

Figure 1 The geographic map of sampling sites in Xinyang city of central China. (Sampling sites 1-4 located in Shihe District and sampling sites 5-8 located in Luoshan County). 
Table 1 Comparison of the differences in the positive rate of Theileria spp. in the sheep and the hedgehogs in the different locations

\begin{tabular}{|c|c|c|c|c|c|c|c|c|}
\hline Location & Animal species & Number sampled & Number positive & Positive rate & $x^{2}$ & DF & RR (95\% Cl) & $\mathbf{P}$ \\
\hline \multirow{2}{*}{ Shihe } & Hedgehogs & 51 & 19 & $37.25 \%$ & \multirow{2}{*}{1.97} & \multirow{2}{*}{1} & 1.00 & \multirow{2}{*}{0.160} \\
\hline & Sheep & 53 & 27 & $50.94 \%$ & & & $1.37(0.88-2.13)$ & \\
\hline \multirow{2}{*}{ Luoshan } & Hedgehogs & 101 & 2 & $1.98 \%$ & \multirow{2}{*}{73.71} & \multirow{2}{*}{1} & 1.00 & \multirow{2}{*}{0.000} \\
\hline & Sheep & 20 & 15 & $75.00 \%$ & & & $37.88(9.38-152.87)$ & \\
\hline \multirow{2}{*}{ Total } & Hedgehogs & 152 & 21 & $13.82 \%$ & \multirow{2}{*}{46.75} & \multirow{2}{*}{1} & 1.00 & \multirow{2}{*}{0.0000} \\
\hline & Sheep & 73 & 42 & $57.53 \%$ & & & $4.16(2.67-6.49)$ & \\
\hline
\end{tabular}

$\mathrm{DF}=$ degrees of freedom; $\mathrm{RR}=$ risk ratio; $\mathrm{Cl}=$ confidence interval.

\section{DNA extraction and PCR amplification}

Total genomic DNA was extracted from the whole blood of each sample using DNeasy Blood \& Tissue Kit (Qiagen, Germany) according to the manufacturer's instructions. Molecular detection of Babesia spp. and Theileria spp. was carried out using a nested PCR protocol targeting the $18 \mathrm{~S}$ ribosomal RNA gene with specific primers in accordance with the method established by Silveira JA et al. [28]. Aliquot of double distilled water was used as the negative control to detect contamination. PCR was carried out in a C1000 Touch $^{\mathrm{Tm}}$ Thermal Cycler (BIORAD, USA). PCR products were separated by electrophoresis in $1.5 \%$ agarose gel. The positive PCR products were sent to Sangon Biotech (Shanghai, China) for sequencing in both directions.

\section{Phylogenetic analysis}

The obtained 18S rRNA gene sequences were edited using the BioEdit 7.0.9 software. The pairwise nucleotide percent identity of the new sequences was calculated using MegAlign software (DNAStar Inc., Madison, WI, USA). The neighbor-joining tree was constructed using the MEGA 5.05 package [29]. Distances were estimated by the Kimura 2-parameter model and the numbers above the branch demonstrate bootstrap support from 1000 replications. The $18 \mathrm{~S}$ rRNA gene sequence of Cryptosporidium fragile (EU1627541) was included in the tree as an outgroup.

\section{Statistical analysis}

Differences in the positive rates of Theileria spp. in domestic and wild animals were tested by $\chi^{2}$-test, which was performed in SPSS 18.0.

\section{Results}

Detection of the Babesia spp. and the Theileria spp. infections in animals

Out of 227 samples, 63 (21 hedgehogs and 42 sheep) were detected positive for Theileria spp. in the two regions. Babesia spp. was only detected in the two dogs.
The positive rate of Theileria spp. in the hedgehogs and sheep in Shihe District were 37.25\% and 50.94\%, respectively (see Table 1). There was no statistically significant difference in the positive rate of Theileria spp. in the hedgehogs and sheep in Shihe District $(P>0.05)$. However, there was statistically significant difference in the positive rate of Theileria spp. in the hedgehogs and sheep in Luoshan County. The positive rate of Theileria spp. in the hedgehogs and sheep in Luoshan County was $1.98 \%$ and $75.00 \%$, respectively. The positive rate of Theileria spp. in the sheep was 37.88 times $\left(\chi^{2}=73.71\right.$, $\mathrm{DF}=1, P<0.05)$ higher than that in the hedgehogs. The total positive rate of Theileria spp. in the hedgehogs and sheep in these two regions were $13.82 \%$ and $57.53 \%$, respectively. The total positive rate of Theileria spp. in the sheep in these two regions was 4.16 times $\left(\chi^{2}=46.75\right.$, $\mathrm{DF}=1, P<0.05$ ) higher than that in the hedgehogs (see Table 1). There was no statistically significant difference in the positive rate of Theileria spp. in the sheep between the two regions. However, the positive rate of Theileria spp. in the hedgehogs in Shihe District was 18.81 times $\left(\chi^{2}=35.41, \mathrm{DF}=1, P<0.05\right)$ higher than that in Luoshan County (see Table 2).

\section{Sequencing and phylogenetic analysis}

T. luwenshuni was identified in 61 samples, 15 sheep and two hedgehogs in Luoshan County, and 26 sheep and 18 hedgehogs in Shihe District. Theileria sp. was detected in one hedgehog and one sheep respectively in Shihe District. B. gibsoni was identified in the two dogs in Shihe District. The sequences of detected piroplasms in this study were deposited into GenBank. The accession numbers are KJ715184, KJ715185, KJ715188, KJ715189, KJ715191, KJ715192, KJ715193, KJ715178 and KJ715179 (see Figure 2).

The obtained sequences were edited and assembled to a final length of 350 base pairs (bp). All the Theileria spp. detected in this study had 94.6\%-99.7\% identity with nucleotide differences of 1-19 bp (see Table 3). The constructed phylogenetic tree of maximum-likelihood analysis depicting the relationships of the $18 \mathrm{~S}$ rRNA 
Table 2 Comparison of the differences in the positive rate of Theileria spp. in the sheep and hedgehog in Luoshan and Shihe

\begin{tabular}{|c|c|c|c|c|c|c|c|c|}
\hline Animal species & Location & Number sampled & Number positive & Positive rate & $x^{2}$ & DF & $\mathrm{RR}(95 \% \mathrm{Cl})$ & $\mathbf{P}$ \\
\hline \multirow{2}{*}{ Sheep } & Luoshan & 20 & 15 & $75.00 \%$ & \multirow{2}{*}{3.44} & \multirow{2}{*}{1} & 1.00 & \multirow{2}{*}{0.0636} \\
\hline & Shihe & 53 & 27 & $50.94 \%$ & & & $0.68(0.47-0.98)$ & \\
\hline \multirow{2}{*}{ Hedgehogs } & Luoshan & 101 & 2 & $1.98 \%$ & \multirow{2}{*}{35.41} & \multirow{2}{*}{1} & 1.00 & \multirow{2}{*}{0.0000} \\
\hline & Shihe & 51 & 19 & $37.25 \%$ & & & $18.81(4.56-77.65)$ & \\
\hline
\end{tabular}

$\mathrm{DF}=$ degrees of freedom; $\mathrm{RR}=$ risk ratio; $\mathrm{Cl}=$ confidence interval.

gene of the Theileria and Babesia species identified in this study and those present in the GenBank database is shown in Figure 2. The sequences of Theileria spp. in this study fall into the same clade with T. lunwenshuni (KC854408) cloned from a small ruminant in China and Theileria sp. (HQ844673) isolated from a febrile hospitalized patient in China (see Figure 2). The sequences of Babesia spp. are in the same clade with B. gibsoni (FJ769386) cloned from a dog in Taiwan.

\section{Discussion}

In this study, we found that $27.11 \%(61 / 225)$ samples from sheep and hedgehogs were positive for T. luwenshuni. This result suggests that T. luwenshuni was the predominant Theileria species in both hedgehogs and sheep in the two studied regions. This study is the first to report on the identification of T. luwenshuni in small wild mammals in Central China. Other Theileria species, e.g. T. uilenbergi and T. ovis, have been reported in the southeastern parts of China [30], but were not found in this study. This may possibly be associated with the ecosystem features of the local environment.

It was reported that $T$. lunwenshuni was transmitted by Haemaphysalis qinghaiensis [31], and small ruminant animals were infected with it in the northern and central parts of China [32], with high pathogenicity causing great economic losses. Animals that survived acute infection could become low-level carriers of the parasite,

Table 3 Nucleotide percent identity between the 18S rRNA of Theileria spp. detected in this study

\begin{tabular}{|c|c|c|c|c|c|c|c|}
\hline \multicolumn{2}{|c|}{ Sequence } & \multirow[t]{2}{*}{1} & \multirow[t]{2}{*}{2} & \multirow[t]{2}{*}{3} & \multirow[t]{2}{*}{4} & \multirow[t]{2}{*}{5} & \multirow[t]{2}{*}{6} \\
\hline 1 & KJ715184 & & & & & & \\
\hline 2 & KJ715185 & 96.3 & & & & & \\
\hline 3 & KJ715188 & 98.9 & 96.3 & & & & \\
\hline 4 & KJ715189 & 98.9 & 96.0 & 98.9 & & & \\
\hline 5 & KJ715191 & 98.6 & 95.4 & 98.9 & 98.3 & & \\
\hline 6 & KJ715192 & 98.6 & 94.6 & 98.6 & 98.9 & 98.3 & \\
\hline 7 & KJ715193 & 98.9 & 96.3 & 99.7 & 98.9 & 99.1 & 99.1 \\
\hline
\end{tabular}

and would remain persistently infected for years without apparent clinical signs [33]. In the present study, all the animals positive for $T$. lunwenshuni did not show any clinical symptoms or signs of the disease. However, if vector ticks feed on these sub-clinical carriers, they can be infected and transmit the pathogen to exotic animals [33], and even to human beings.

One exciting finding of this study was, as revealed by the phylogenetic analysis, that the Theileria spp. detected in this study was very close to the Theileria sp. isolated from a febrile hospitalized patient in Suizhou city of Hubei province. In addition, Suizhou city is adjacent to Shihe District geographically. Thus, whether the pathogens were different strains of the same species, whether they would be pathogenic to different species of host animals, or whether they could be a potential threat to human beings is still unclear, and this finding warrants further studies.

In this epidemiological study on piroplasms infection, it is important to understand the role of the different host animals in the transmission cycle [34-36]. We found that the total positive rate of Theileria spp. in the sheep in the two study regions was much higher than that in the hedgehogs, indicating that domestic animals might play a more essential role in the transmission of piroplasmosis. This result was supported by the following facts: First, the population density of domestic animals is much higher than that of wild animals. In fact, sheep husbandry is very common in the rural areas of the study regions. A total of 818.4 thousands of sheep were raised in Xinyang city, based on the data from the Henan Statistical Yearbook 2012. These huge numbers of sheep posed a high risk of exposure and infection to livestock, and increase the economic burden on animal husbandry and farmers in these regions. Second, the habits of animals are indicative of their exposures to infections. Although the population size of hedgehogs in these regions is unavailable, the living habits of hedgehogs are definitely different from domestic animals as hedgehogs are not gregarious animals [37-40]. This may reduce the risk of exposure to tick vectors. Moreover, the positive rate of Theileria spp. in the hedgehogs in 


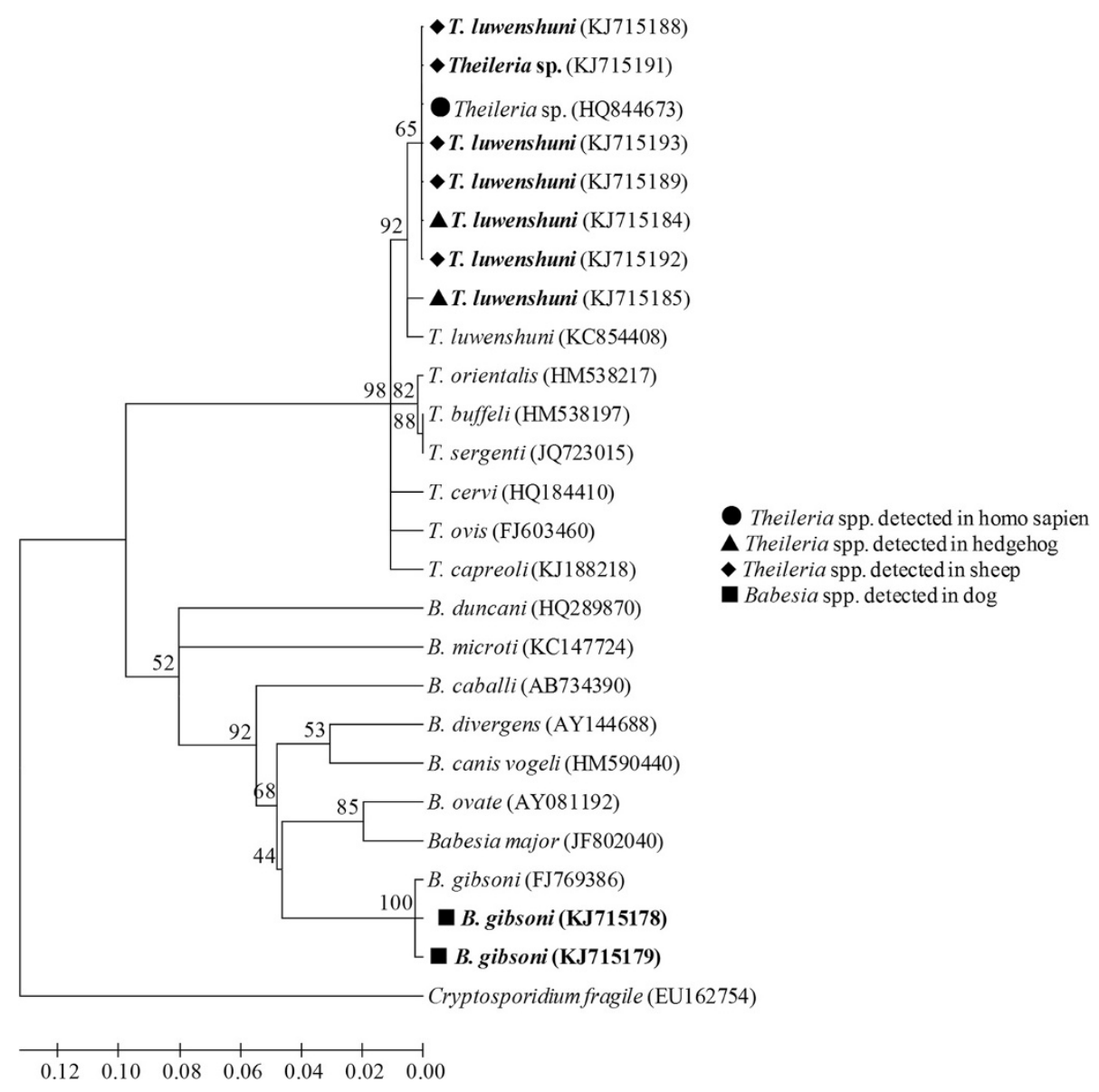

Figure 2 Neighbor-joining tree showing the phylogenetic relationship of the $18 \mathrm{~S}$ rRNA gene sequences of Theileria and Babesia species identified in this study and those present in the GenBank database. (The GenBank accession numbers are indicated in parentheses. Species detected in this study are indicated in bold. The scale bar represents nucleotide substitutions per position).

Shihe District was much higher than that in Luoshan County, it also indicated that the population density of sheep in Shihe District might be much higher than that in Luoshan County. Sheep might live in closer contact with hedgehogs in Shihe District due to the increased environmental and social economic activities.

In this study, Babesia spp. was only detected in dogs. This could be attributed to only one transmission season, as well as to the limited number of host species that was taken into account. However, babesiosis had been reported in livestock in Henan province [41]. Previous studies on ticks [42] and rodents [43] collected in Xinyang city documented the detection of $B$. microti, which could cause babesiosis in animals and humans. Therefore, it is necessary to carry out more studies on the epidemiology of Babesia spp. and Theileria spp. infections in more species of domestic and wild animals, as well as on the infection status of vector ticks in these regions.

\section{Conclusion}

Babesia spp. and Theileria spp. infections were detected in both domestic and wild animals in the study regions of Central China. Further studies are needed to estimate the impacts to local animal husbandry by piroplasms infection and to establish biological measures to control the vector ticks.

\section{Additional file}

Additional file 1: Multilingual abstracts in the six official working languages of the United Nations.

\section{Competing interests}

The authors declare that they have no competing interests.

\section{Authors' contributions}

ZC conducted the field sampling, performed the laboratory work, generated experimental data, and wrote the manuscript. QL, X-NZ and B-LX had a substantial role in the conception of the study, guidance of the practical 
work and writing of the manuscript. F-CJ helped with the sample collection. All authors read and approved the final manuscript.

\section{Acknowledgements}

This work was supported by the Special Fund for Health Research in the Public Interest (Grant No. 201202019).

\section{Author details}

${ }^{1}$ National Institute of Parasitic Diseases, Chinese Center for Disease Control and Prevention, Shanghai 200025, People's Republic of China. 'WHO Collaborative Center for Malaria, Schistosomiasis and Filariasis; Key Laboratory of Parasite and Vector Biology, Ministry of Health, Shanghai 200025, People's Republic of China. ${ }^{3}$ Xinyang College of Agriculture and Forestry, Xinyang 464000, People's Republic of China. ${ }^{4}$ Henan Center for Disease Control and Prevention, Zhengzhou 450016, People's Republic of China.

Received: 18 April 2014 Accepted: 27 May 2014

Published: 3 June 2014

\section{References}

1. Uilenberg G: Babesia-A historical overview. Vet Parasitol 2006, 138:3-10.

2. Schnittger $L$, Rodriguez AE, Florin-Christensen M, Morrison DA: Babesia: a world emerging. Infect Genet Evol 2012, 12:1788-1809.

3. He DM, Wang YM: Progress in research of human babesiosis. Chin J Infect Dis 2012, 30:638-640.

4. Xu Y, Zhang S, Huang X, Bayin C, Xuan X, Igarashi I, Fujisaki K, Kabeya H, Maruyama S, Mikami T: Seroepidemiologic studies on Babesia equi and Babesia caballi infections in horses in Jilin province of China. J Vet Med Sci 2003, 65:1015-1017.

5. Xuan X, Chahan B, Huang X, Yokoyama N, Makala LH, Igarashi I, Fujisaki K, Maruyama S, Sakai T, Mikami T: Diagnosis of equine piroplasmosis in Xinjiang province of China by the enzyme-linked immunosorbent assays using recombinant antigens. Vet Parasitol 2002, 108:179-182.

6. Xie J, Liu G, Tian Z, Luo J: Development of loop-mediated isothermal amplification (LAMP) for detection of Theileria equi. Acta Trop 2013, 127:245-250.

7. He L, Feng HH, Zhang WJ, Zhang QL, Fang R, Wang LX, Tu P, Zhou YQ, Zhao JL, Oosthuizen MC: Occurrence of Theileria and Babesia species in water buffalo (Bubalus babalis, Linnaeus, 1758) in the Hubei province, South China. Vet Parasitol 2012, 186:490-496.

8. Liu Q, Zhou YQ, He GS, Oosthuizen MC, Zhou DN, Zhao JL: Molecular phylogenetic studies on Theileria spp. isolates (China) based on small subunit ribosomal RNA gene sequences. Trop Anim Health Prod 2010, 42:109-114.

9. Liu AH, Guan GQ, Liu ZJ, Liu JL, Leblanc N, Li YQ, Gao JL, Ma ML, Niu QL, Ren QY, Bai Q, Yin H, Luo JX: Detecting and differentiating Theileria sergenti and Theileria sinensis in cattle and yaks by PCR based on major piroplasm surface protein (MPSP). Exp Parasitol 2010, 126:476-481.

10. Yin H, Schnittger L, Luo J, Seitzer U, Ahmed JS: Ovine theileriosis in China: a new look at an old story. Parasitol Res 2007, 101(Suppl 2):191-195.

11. Yin H, Liu Z, Guan G, Liu A, Ma M, Ren Q, Luo J: Detection and differentiation of Theileria luwenshuni and Theileria uilenbergi infection in small ruminants by PCR. Transbound Emerg Dis 2008, 55:233-237.

12. Liu AH, Yin H, Guan GQ, Schnittger L, Liu ZJ, Ma ML, Dang ZS, Liu JL, Ren QY, Bai Q, Ahmed JS, Luo JX: At least two genetically distinct large Babesia species infective to sheep and goats in China. Vet Parasitol 2007, 147:246-251.

13. Niu Q, Luo J, Guan G, Ma M, Liu Z, Liu A, Dang Z, Gao J, Ren Q, Li Y, Liu J, Yin H: Detection and differentiation of ovine Theileria and Babesia by reverse line blotting in China. Parasitol Res 2009, 104:1417-1423.

14. Shen Y, Gao J, Xu K, Xue L, Zhang Y, Shi B, Li D, Wei X, Higuchi S: Babesiasis in Nanjing area, China. Trop Anim Health Prod 1997, 29(Suppl 4):19-22.

15. Wei FR, Lan QX, Zhu D, Ye JH, Liu Q, Zhang Y: Investigation on Babesia in ticks infested on police dogs in selected areas of China. Chin J Parasitol Parasit Dis 2012, 30:390-392.

16. Zhou X, Li SG, Chen SB, Wang JZ, Xu B, Zhou HJ, Ge HX, Chen JH, Hu W: Co-infections with Babesia microti and Plasmodium parasites along the China-Myanmar border. Infect Dis Poverty 2013, 2:24.
17. Yao LN, Ruan W, Zeng CY, Li ZH, Zhang X, Lei YL, Lu QY, Che HL: Pathogen identification and clinical diagnosis for one case infected with Babesia. Chin J Parasitol Parasit Dis 2012, 30:118-121.

18. Fan DH, Li M, Xu HF, Hu MX, Zhang J, Sun Y: The situation of mice and ticks infected by Babesia microti. Chin J Hyg Insect Equip 2012, 1:48-50.

19. Wang ZS, Wang XM, Dou XF, Li X, Ren HL, Liu J, Bu LY, Wang H: First report of Babesia microti infection in rodents in Miyun district of Beijing. Acta Parasitol Med Entomol Sin 2013, 20:218-222.

20. Jiang LP, Zhang L, Bao QH, Lu QY, Cheng SY, Xu BX: Babesia DNA segments detected from rodents in Zhejiang province. Chin J Vector Biol Control 2012, 23:303-305.

21. Van Peenen PF, Chang SJ, Banknieder AR, Santana FJ: Piroplasms from Taiwanese rodents. J Protozool 1977, 24:310-312.

22. He L, Khan MK, Zhang WJ, Zhang QL, Zhou YQ, Hu M, Zhao J: Detection and identification of Theileria infection in sika deer (Cervus nippon) in China. J Parasitol 2012, 98:598-603.

23. Brown CG, Ilhan T, Kirvar E, Thomas M, Wilkie G, Leemans I, Hooshmand-Rad P: Theileria lestoquardi and Theileria annulata in cattle, sheep, and goats In vitro and in vivo studies. Ann N Y Acad Sci 1998, 849:44-51.

24. Kawazu S, Kamio T, Kakuda T, Terada Y, Sugimoto C, Fujisaki K: Phylogenetic relationships of the benign Theileria species in cattle and Asian buffalo based on the major piroplasm surface protein ( $p 33 / 34)$ gene sequences. Int J Parasitol 1999, 29:613-618.

25. Ueti MW, Mealey RH, Kappmeyer LS, White SN, Kumpula-McWhirter N, Pelzel AM, Grause JF, Bunn TO, Schwartz A, Traub-Dargatz JL, Hendrickson A, Espy B, Guthrie AJ, Fowler WK, Knowles DP: Re-emergence of the apicomplexan Theileria equi in the United States: elimination of persistent infection and transmission risk. PLoS One 2012, 7:e44713.

26. Gabrielli S, Calderini P, Cassini R, Galuppi R, Tampieri MP, Pietrobelli M, Cancrini G: Human exposure to piroplasms in Central and Northern Italy. Vet Ital 2014, 50:41-47.

27. NCBI Nucleotide Database: [http://www.ncbi.nlm.nih.gov/nuccore/339655465]

28. Silveira JA, Rabelo ÉM, Lacerda AC, Borges PA, Tomás WM, Pellegrin AO, Tomich RG, Ribeiro MF: Molecular detection and identification of hemoparasites in pampas deer (Ozotoceros bezoarticus Linnaeus, 1758) from the Pantanal Brazil. Ticks Tick Borne Dis 2013, 4:341-345.

29. Tamura K, Peterson D, Peterson N, Stecher G, Nei M, Kumar S: MEGA5: molecular evolutionary genetics analysis using maximum likelihood, evolutionary distance, and maximum parsimony methods. Mol Biol Evol 2011, 28(10):2731-2739.

30. Zhang X, Liu Z, Yang J, Chen Z, Guan G, Ren Q, Liu A, Luo J, Yin H, Li Y: Multiplex PCR for diagnosis of Theileria uilenbergi, Theileria luwenshuni, and Theileria ovis in small ruminants. Parasitol Res 2014, 113:527-531.

31. Niu Q, Guan G, Liu Z, Ma M, Li Y, Liu A, Ren Q, Liu J, Luo J, Yin H: Simultaneous detection of piroplasma infections in field Haemaphysalis qinghaiensis ticks by reverse line blotting. Exp Appl Acarol 2012, 56:123-132.

32. Ge Y, Pan W, Yin H: Prevalence of Theileria infections in goats and sheep in southeastern China. Vet Parasitol 2012, 186:466-469.

33. Neitz WO: Classification, transmission, and biology of piroplasms of domestic animals. Ann N Y Acad Sci 1956, 64:56-111.

34. Li Y, Zhang X, Liu Z, Chen Z, Yang J, He H, Guan G, Liu A, Ren Q, Niu Q, Liu J, Luo J, Yin H: An epidemiological survey of Theileria infections in small ruminants in central China. Vet Parasitol 2014, 200:198-202.

35. Boldbaatar D, Xuan X, Battsetseg B, Igarashi I, Battur B, Batsukh Z, Bayambaa $B$, Fujisaki K: Epidemiological study of equine piroplasmosis in Mongolia. Vet Parasitol 2005, 127:29-32.

36. Moretti A, Mangili V, Salvatori R, Maresca C, Scoccia E, Torina A, Moretta I, Gabrielli S, Tampieri MP, Pietrobelli M: Prevalence and diagnosis of Babesia and Theileria infections in horses in Italy: a preliminary study. Vet J 2010, 184:346-350.

37. Dierenfeld ES: Feeding behavior and nutrition of the african pygmy hedgehog (Atelerix albiventris). Vet Clin North Am Exot Anim Pract 2009, 12:335-337.

38. Simone-Freilicher EA, Hoefer HL: Hedgehog care and husbandry. Vet Clin North Am Exot Anim Pract 2004, 7:257-267.

39. Teng Z, Liu C, Lin Y: Ecological habits, breeding and utilization of Erinaceus europaeus. Yantai Normal University Journal (Natural Science Edition) 2000, 17:131-133.

40. Wen-xiang MA: A study on ecological habit of hedgehog in Qufu Konglin. J Biol 2001, 4:11. 
41. Liu J, Yin H, Liu G, Guan G, Ma M, Liu A, Liu Z, Li Y, Ren Q, Dang Z, Gao J, Bai Q, Zhao H, Luo J: Discrimination of Babesia major and Babesia ovata based on ITS1-5.8S-ITS2 region sequences of rRNA gene. Parasitol Res 2008, 102:709-713.

42. Chen Z, Liu Q, Liu JQ, Xu BL, Lv S, Xia S, Zhou XN: Tick-borne pathogens and associated co-infections in ticks collected from domestic animals in central China. Parasit Vectors 2014, 7:237.

43. Zhao XG, Li H, Sun Y, Zhang YY, Jiang JF, Liu W, Cao WC: Dual infection with Anaplasma phagocytophilum and Babesia microti in a Rattus norvegicus, China. Ticks Tick Borne Dis 2013, 4:399-402.

doi:10.1186/2049-9957-3-18

Cite this article as: Chen et al:: Detection of piroplasms infection in sheep, dogs and hedgehogs in Central China. Infectious Diseases of Poverty 2014 3:18.

\section{Submit your next manuscript to BioMed Central and take full advantage of:}

- Convenient online submission

- Thorough peer review

- No space constraints or color figure charges

- Immediate publication on acceptance

- Inclusion in PubMed, CAS, Scopus and Google Scholar

- Research which is freely available for redistribution 